\title{
Relaciones biogeográficas de los grandes ríos de la cuenca del Plata basadas en ensambles de serpientes
}

\author{
Biogeographical relationships of the large rivers of the Plata Basin based on snake assemblages \\ Vanesa Arzamendia ${ }^{\mathrm{a}, \mathrm{b}, *}$, Alejandro R. Giraudo ${ }^{\mathrm{a}, \mathrm{b}}$ y Gisela P. Bellini ${ }^{\mathrm{a}}$ \\ a Instituto Nacional de Limnología, Consejo Nacional de Investigaciones Científicas y Tecnológicas-Universidad Nacional del Litoral, Ciudad Universitaria, \\ 3000 Santa Fe, Argentina \\ ${ }^{\mathrm{b}}$ Facultad de Humanidades y Ciencias, Universidad Nacional del Litoral, Ciudad Universitaria, 3000 Santa Fe, Argentina
}

Recibido el 8 de enero de 2015; aceptado el 9 de abril de 2015

Disponible en Internet el 20 de agosto de 2015

\begin{abstract}
Resumen
Los grandes ríos del Plata fluyen hacia el sur desde latitudes intertropicales a templadas y extienden la distribución de especies tropicales. Examinamos las siguientes hipótesis sobre relaciones biogeográficas de la ofidiofauna entre tramos de los ríos: el Alto Paraná tiene mayor afinidad con el Alto Uruguay, el Paraná Medio con el Paraguay Inferior respecto otros tramos del propio Paraná, y el Delta Superior tiene mayor afinidad con el Paraná Medio y el Delta Inferior con el Uruguay Inferior. Comparamos los tramos de los ríos y estos con las unidades fitogeográficas, usando 13,039 ejemplares de 104 taxones, mediante análisis de parsimonia de endemismos y similitud. Los resultados apoyan las 2 primeras hipótesis, no la tercera. El Delta Superior-Delta Inferior formaron un grupo con elevada similitud relacionado con el Uruguay Medio-Uruguay Inferior, y con el análisis de parsimonia de endemismos formaron un clado separado. Los tramos se relacionaron con las biorregiones que atraviesan, aunque el Uruguay Medio-Inferior solo con la provincia Paranaense (análisis de parsimonia de endemismos), debido a especies atlántico-paranaenses que alcanzan mayores latitudes por este río. El Paraná Medio-Paraguay Inferior-Paraná Superior se relacionan con el Chaco Húmedo y se discuten criterios de regionalización previos. Los ríos del Plata constituyen áreas de transición biogeográfica importantes para su conservación debido a la superposición de componentes bióticos tropicales y templados.

Derechos Reservados @ 2015 Universidad Nacional Autónoma de México, Instituto de Biología. Este es un artículo de acceso abierto distribuido bajo los términos de la Licencia Creative Commons CC BY-NC-ND 4.0.
\end{abstract}

Palabras clave: Análisis de parsimonia de endemismos; Análisis multivariados; Serpientes; Ríos de América del Sur; Transición biogeográfica; Argentina

\section{Abstract}

The large rivers of the Plata flow southward from intertropical to temperate latitudes, extending the distribution of tropical species. We tested biogeographic hypotheses about relationships among sections of the rivers comparing their snake fauna: the High Paraná has greater affinity with the High Uruguay, the Middle Paraná with Lower Paraguay regarding other sections of Paraná itself, and the Upper Delta has greater affinity with the Middle Paraná and the Lower Delta with Lower Uruguay. We compared sections of rivers and these with phytogeographical units, by means of 13,039 records of 104 taxa, using parsimony analysis of endemicity and similarity. Our results support the first two hypotheses, not the third one. Upper Delta-Lower Delta formed a group with high similarity related to the Middle Uruguay-Lower Uruguay, and with PAE it formed a separate clade. Sections were associated with the bioregions that they crossed, although the Middle-Lower Uruguay did it only with the Parana

\footnotetext{
* Autor para correspondencia.

Correo electrónico: vanearzamendia@gmail.com (V. Arzamendia).

La revisión por pares es responsabilidad de la Universidad Nacional Autónoma de México.
} 
province (parsimony analysis of endemicity) due to Atlantic-Parana species reaching higher latitudes by this river. The Middle Paraná-Lower Paraguay-Upper Paraná are related to Humid Chaco, being discussed previous published criteria of regionalization. Because of the overlap of tropical and temperate biotic components, larger Plata Rivers are biogeographic transition areas that are relevant to be preserved.

All Rights Reserved @ 2015 Universidad Nacional Autónoma de México, Instituto de Biología. This is an open access item distributed under the Creative Commons CC License BY-NC-ND 4.0.

Keywords: Parsimony analysis of endemicity; Multivariate analysis; Snakes; South American rivers; Biogeographic transition; Argentina

\section{Introducción}

La cuenca del Plata es el segundo sistema fluvial de América del Sur en caudal y representa una región con elevada diversificación biótica que incluye los remanentes más australes del hotspot de la Selva Atlántica, una de las ecorregiones más biodiversas y amenazadas del mundo (Arzamendia y Giraudo, 2012; Galindo-Leal y Câmara, 2003). Los principales recolectores de la cuenca, los ríos Paraná, Paraguay y Uruguay, nacen en latitudes intertropicales y se desplazan hacia latitudes templadas, donde contienen una biota tropical que alcanza mayores latitudes siguiendo su curso (Arzamendia y Giraudo, 2009). El efecto de los grandes ríos de la cuenca del Plata en los patrones de distribución de especies tropicales fue mencionado por diversos autores para distintos taxones de plantas y animales. Por ejemplo, Bonetto (1961) lo indica para náyades de moluscos; Bonetto y Drago (1968) para poríferos, briozoarios y moluscos lamelibranquios; Rabinovich y Rapoport (1975) y Nores, Cerana y Serra (2005) para aves; Ringuelet (1975) para peces; Gallardo (1979) y Gudynas, Williams y Azpelicueta (1988) para anfibios; Cabrera y Willink (1980) para mariposas y mamíferos; José de Paggi (1990) para rotíferos; Morrone y Lopretto (1994) para crustáceos decápodos; Cabrera (1994); Menalled y Adámoli (1995) y Oakley, Prado y Adámoli (2005) para la vegetación; Cei (1993) para reptiles; Giraudo (2001) para serpientes; y Giraudo y Arzamendia (2004) para vertebrados amniotas. Resulta notable que diferentes tramos del mismo río presenten especies de diferentes linajes tropicales y que se observen mayores afinidades biogeográficas con otros ríos que entre sectores del mismo curso (ver detalles en Arzamendia y Giraudo, 2009; Giraudo y Arzamendia, 2004; Menalled y Adámoli, 1995).

Bonetto (1961) y Bonetto y Drago (1968), en trabajos precursores sobre la temática, postularon las siguientes 4 hipótesis sobre las afinidades faunísticas entre los ríos y sus distintos tramos: 1) la fauna del Alto Paraná presenta claras afinidades con la del Uruguay y la de los ríos de la pendiente Atlántica del sur del Brasil; incluidos en la provincia biogeográfica Paranaense y/o Atlántica; 2) existe continuidad faunística entre el eje potámico Paraguay-Paraná Medio, con la desaparición de unas pocas especies hacia el sur; 3) hay influencia amazónica en el río Paraguay Inferior; 4) muchas especies del Alto Paraná no alcanzan el Paraná Medio. En coincidencia con estas hipótesis, Arzamendia y Giraudo (2009) registraron especies de serpientes con distribución amazónica que habitan principalmente en el río Paraguay Inferior y Paraná Medio, mientras que especies atlántico-paranaenses se distribuyen en latitudes meridionales, principalmente por el río Uruguay. Adicionalmente, mostraron que la biota de los ríos de la cuenca del Plata presenta diferencias en la composición de serpientes en diferentes tramos en relación con factores históricos (cambios de relación entre tramos de los ríos y sus paleocauces), ecológicos y geográficos. Observaron que a pesar de la conexión entre el Alto Paraná y el Paraná Medio, un corredor hídrico que facilita la expansión de especies, muchos taxones no alcanzan latitudes australes a través de este corredor fluvial.

Menalled y Adámoli (1995) realizaron un análisis biogeográfico de árboles y arbustos en el Delta del Paraná, concluyendo que la riqueza florística puede ser entendida como consecuencia de la eficiencia diferencial de los ríos Paraná y Uruguay como corredores para especies tropicales hacia latitudes templadas. Propusieron al río Paraná como el principal camino de colonización de la porción norte o superior del Delta, mientras que el río Uruguay cumple un papel similar con el Delta Inferior (DI). Estos autores le atribuyeron al río Uruguay ser la principal ruta de migración de elementos tropicales que conforman la rica comunidad del Bajo Delta, a pesar de que el Paraná ha tenido una mayor preponderancia en la formación geomorfológica de todo el Delta.

Los grandes ríos de la cuenca del Plata contienen componentes bióticos tropicales (del Pantanal, amazónicos o paranaense-atlánticos) que alcanzan latitudes templadas, y se superponen durante sus recorridos con componentes de biotas chaqueñas, del Espinal y pampeanas. Las regiones donde se juntan componentes bióticos de diferente origen se denominan zonas de transición (Halffter, 1978). Estas zonas representan eventos de hibridación biótica, promovidos por cambios ecológicos e históricos y condiciones ambientales, que permiten la mezcla de diferentes componentes bióticos (Ferro y Morrone, 2014; Morrone, 2004). Las afinidades biogeográficas de los taxones son la información más fundamental a tener en cuenta para analizar zonas de transición biogeográficas con precisión (Ferro y Morrone, 2014).

Las afinidades bióticas entre tramos de ríos no han sido analizadas cuantitativamente hasta el presente. Considerando los antecedentes discutidos, este aporte tiene por objetivo poner a prueba las siguientes 3 hipótesis de afinidad faunística entre los distintos tramos de los ríos, comparando sus ensambles de serpientes: 1) el Alto Paraná presenta mayor afinidad con el Alto Uruguay respecto a otros tramos del Paraná (Medio y Delta); 2) el Paraná Medio tiene mayor afinidad con el Paraguay Inferior respecto de otros tramos del propio río Paraná; 3) los ensambles de serpientes del Delta Superior (DS) tienen mayor afinidad con el Paraná Medio, mientras que los del DI tienen mayor afinidad con el río Uruguay Inferior. Adicionalmente, se evalúa si los ensambles de serpientes de los tramos de los ríos se relacionan 

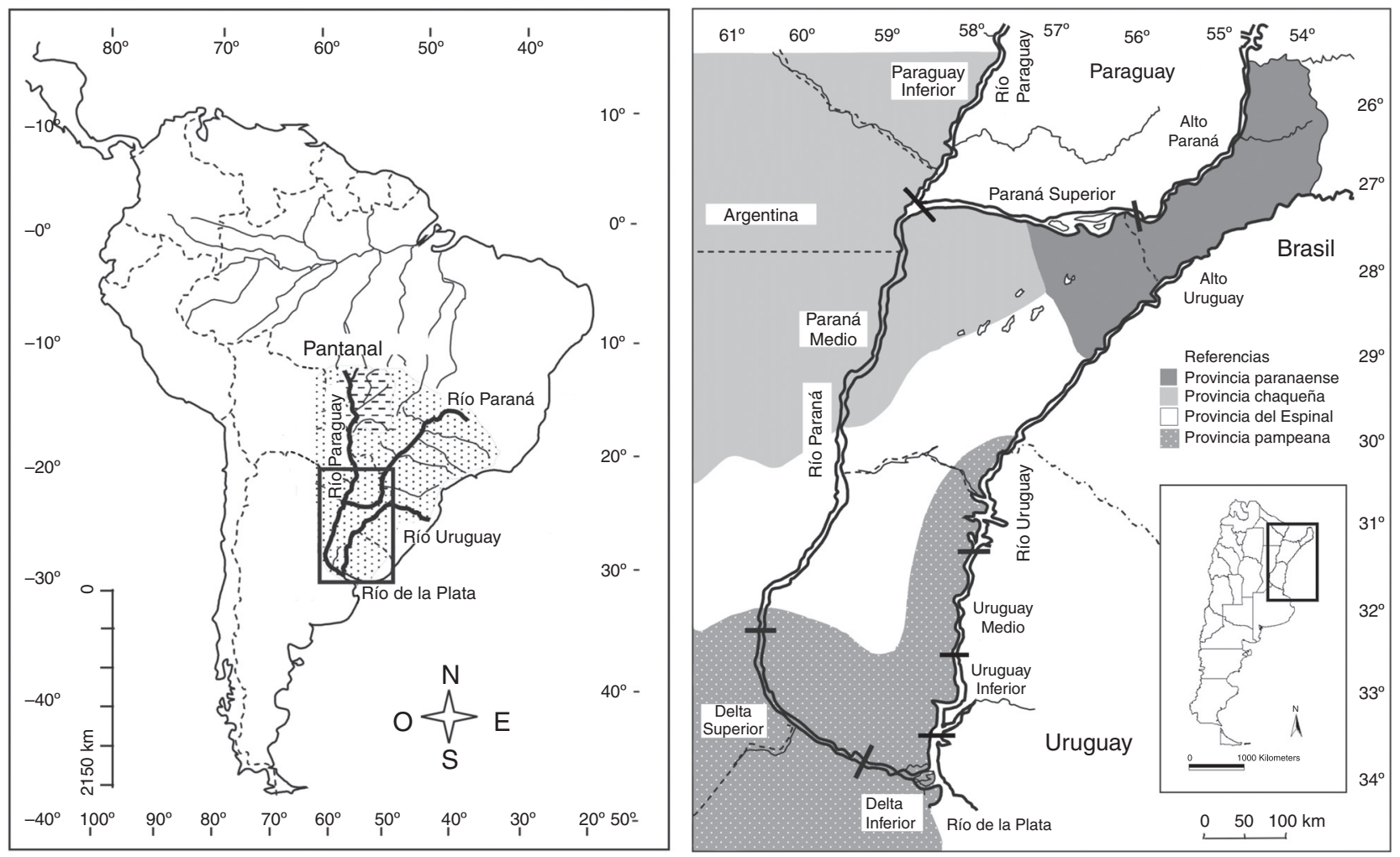

Figura 1. Área de estudio mostrando los grandes ríos de la cuenca del Plata en América del Sur (izquierda) y los tramos de los ríos Paraná, Uruguay y Paraguay, separados por una línea transversal negra; y las regiones fitogeográficas comparadas (derecha).

con las áreas biogeográficas con las que limitan y constituyen zonas de transición biogeográfica.

\section{Materiales y métodos}

Constituida por la región subtropical-templada de la cuenca del Plata, que se extiende entre los 25 y $35^{\circ} \mathrm{S}$, presenta 3 grandes ríos colectores: Paraná, Uruguay y Paraguay (fig. 1). Abarca una superficie de unos $710,000 \mathrm{~km}^{2}$, en el noreste de la Argentina, con un clima que varía desde subtropical húmedo en el norte, a templado húmedo de llanura en el sur. Las temperaturas medias anuales varían desde $\operatorname{los} 22^{\circ} \mathrm{C}$ en el norte a los $17^{\circ} \mathrm{C}$ al sur, y las precipitaciones medias anuales desde $1,800 \mathrm{~mm}$ (Misiones) a 1,000 mm (Santa Fe, Entre Ríos y Buenos Aires) (Paoli, Iriondo y García, 2000). Según Cabrera (1994) la región se enmarca en las siguientes provincias fitogeográficas (fig. 1): 1) Paranaense: selvas húmedas subtropicales pluriestratificadas y semideciduas; 2) Chaqueña: representada por el distrito Chaqueño Oriental o Chaco Húmedo, caracterizado por bosques xerófilos de quebracho colorado (Schinopsis balansae), palmares de Copernicia alba y sabanas; 3) del Espinal: bosques xerófilos caducifolios dominados por Prosopis, palmares de Butia yatay, sabanas graminosas y estepas arbustivas; 4) Pampeana: estepas de gramíneas.

Los tramos de los ríos fueron zonificados de acuerdo con sus características geomorfológicas e hidrológicas (Arzamendia y Giraudo, 2009; Bonetto, 1986; Di Persia y Neiff, 1986; Menalled y Adámoli, 1995; Paoli et al., 2000) (fig. 1): 1) Alto Paraná: desde la confluencia de los ríos Paraná e Iguazú (Misiones) hasta la población de Santa Ana (Misiones). Estrecho valle de inundación $(0.5-1 \mathrm{~km})$, con afloramientos y muy pocas islas basálticas, y costas elevadas con selva Paranaense y cañaverales de Guadua angustifolia (Matteucci, Morello, Rodríguez y Mendoza, 2004). 2) Paraná Superior: desde Santa Ana (Misiones) hacia el este donde se encontraban las últimas restingas de basaltos de los ya desaparecidos rápidos del Apipé (inundados por la presa de Yacyretá) hasta la confluencia con el Paraguay en Paso de la Patria (Corrientes). Amplia planicie de inundación $(6-30 \mathrm{~km})$, con restingas de basaltos por sectores, costas elevadas con selvas, numerosas islas sedimentarias con lagunas y esteros interiores, selvas ribereñas y en galería, bosques de sauce (Salix humboldtiana) y alisos de río (Tessaria integrifolia). 3) Paraná Medio: desde Paso de la Patria (Corrientes, en la confluencia entre el Paraná y el Paraguay) hasta Diamante (Entre Ríos). Amplia llanura de inundación (6-40 km), con numerosos brazos e islas que contienen muchas lagunas y esteros. Selvas ribereñas empobrecidas, con mayor desarrollo al norte, en las costas poco inundables se observan bosques xerófilos con elementos del Chaco y el Espinal según la latitud. Islas dominadas por especies acuáticas, palustres y bosques fluviales (Lewis y Franceschi, 1979). 4) DS: desde Diamante (Entre Ríos) hasta aproximadamente Puerto Ibicuy (Entre Ríos). Amplia planicie de inundación (60km o más). Costas altas con selva en galería, bosques fluviales de sauces y alisos de río, islas bajas con mayor frecuencia de inundaciones y grandes lagunas con abundante vegetación flotante, palustre (Malvárez, 1999). En barrancas costeras existen bosques xerófilos del Espinal y sabanas. 5) DI: desde Puerto Ibicuy (Entre Ríos) hasta el Río de La Plata 
Tabla 1

Lista de los taxones de serpientes analizados.

\begin{tabular}{|c|c|c|c|c|c|}
\hline 1 & Amerotyphlops brongersmianus & 36 & E. miliaris orinus & 71 & Psomophis genimaculatus \\
\hline 2 & Liotyphlops beui & 37 & E. poecilogyrus caesius & 72 & P. obtusus \\
\hline 3 & L. ternetzii & 38 & E.p. schotti & 73 & Rachidelus brazili \\
\hline 4 & Epictia albipuncta & 39 & E. p. sublineatus & 74 & Sibynomorphus mikanii \\
\hline 5 & E. munoai & 40 & E. reginae & 75 & S. turgidus \\
\hline 6 & E. vellardi & 41 & E. sagittifer & 76 & S. ventrimaculatus \\
\hline 7 & Epicrates crassus & 42 & E. semiaureus & 77 & Taeniophallus occipitalis \\
\hline 8 & Eunectes notaeus & 43 & Helicops infrataeniatus & 78 & T. poecilopogon \\
\hline 9 & Chironius bicarinatus & 44 & H. leopardinus & 79 & Thamnodynastes chaquensis \\
\hline 10 & C. exoletus & 45 & Hydrodynastes gigas & 80 & T. hypoconia \\
\hline 11 & C. maculoventris & 46 & Hydrops caesurus & 81 & T. lanei \\
\hline 12 & Leptophis ahaetulla marginatus & 47 & Imantodes cenchoa & 82 & T. strigatus \\
\hline 13 & Mastigodryas bifossatus bifossatus & 48 & Leptodeira annulata pulchriceps & 83 & Tomodon dorsatus \\
\hline 14 & M. b. triseriatus & 49 & Lygophis anomalus & 84 & T. ocellatus \\
\hline 15 & Spilotes pullatus anomalepis & 50 & L. dilepis & 85 & Xenodon dorbignyi \\
\hline 16 & Tantilla melanocephala & 51 & L. flavifrenatus & 86 & $X$. histricus \\
\hline 17 & Apostolepis assimilis & 52 & L. meridionalis & 87 & $X$. merremii \\
\hline 18 & A. dimidiata & 53 & Oxyrhopus clathratus & 88 & X. neuwiedii \\
\hline 19 & A. quirogai & 54 & O. guibei & 89 & X. pulcher \\
\hline 20 & Atractus paraguayensis & 55 & O. petola & 90 & $X$. semicinctus \\
\hline 21 & A. reticulatus & 56 & O. rhombifer rhombifer & 91 & Micrurus corallinus \\
\hline 22 & A. snethlageae & 57 & Phalotris bilineatus & 92 & M. altirostris \\
\hline 23 & Atractus taeniatus & 58 & P. lemniscatus & 93 & M. baliocoryphus \\
\hline 24 & Boiruna maculata & 59 & P. tricolor & 94 & M. lemniscatus \\
\hline 25 & Clelia clelia & 60 & P. reticulatus & 95 & M. pyrrhocryptus \\
\hline 26 & C. plumbea & 61 & Philodryas agassizii & 96 & M. silviae \\
\hline 27 & Mussurana bicolor & 62 & P. aestiva subcarinata & 97 & Bothrops alternatus \\
\hline 28 & Mussurana quimi & 63 & P. mattogrossensis & 98 & B. cotiara \\
\hline 29 & Paraphimophis rusticus & 64 & P. olfersii latirostris & 99 & B. jararaca \\
\hline 30 & Dipsas bucephala & 65 & P. o. olfersii & 100 & B. jararacussu \\
\hline 31 & Echinanthera cyanopleura & 66 & P. patagoniensis & 101 & B. moojeni \\
\hline 32 & Erythrolamprus aesculapii & 67 & P. psammophidea & 102 & Bothrops cf. neuwiedi \\
\hline 33 & E. almadensis & 68 & Phimophis guerini & 103 & B. diporus \\
\hline 34 & E. frenatus & 69 & Pseudoboa haasi & 104 & Crotalus durissus terrificus \\
\hline 35 & E. jaegeri & 70 & Pseudoeryx plicatilis & & \\
\hline
\end{tabular}

Los números se corresponden con la ubicación en la matriz del anexo 1.

superior (Buenos Aires). Una planicie deltaica con influencia fluvial, islas grandes y bajos inundados, un delta frontal sometido a mareas del Río de La Plata, con islas, albardones y cursos de aguas en forma de abanicos. Islas y costas con selva ribereña y especies de selva Paranaense que faltan en el DS, además de estepas y bosques xerófilos (Kandus, Malvárez y Madanes, 2003). 6) Alto Uruguay: entre los Saltos de Moconá (Misiones) y Concordia (Entre Ríos), con un curso tortuoso con fondo pedregoso y con numerosos rápidos y un valle de inundación relativamente estrecho $(0.5-2 \mathrm{~km})$ con islas de basalto. Costas con barrancas elevadas, con selva Paranaense y cañaverales de Guadua trinii, que se transforma en selva en galería hacia el sur. 7) Uruguay Medio: entre Concordia y Concepción (Entre Ríos). Con menor pendiente y corriente, aunque con más islas que delimitan numerosos brazos, con un ancho entre costa de $1-5 \mathrm{~km}$. Sus costas elevadas y las islas presentan selva con fisonomía Paranaense en galería más diversa que la que se registra en el río Paraná a la misma latitud. También en costas altas se observan bosques xerófilos del Espinal, palmares de Butia y sabanas. 8) Uruguay Inferior: entre Concepción (Entre Ríos) y el DI del río Paraná a $40 \mathrm{~km}$ al sur de Gualeguaychú (Entre Ríos). Se amplía la planicie de inundación del río y comienza la formación de un delta, aunque de fondo arenoso. Con selvas en galería con especies paranaenses, aunque con pérdida de taxones hacia el sur, además de estepas y sabanas con bosques xerófilos del Espinal.9) Paraguay Inferior: entre el límite de Argentina y Paraguay desde Clorinda (Formosa) hasta Paso de La Patria (Corrientes), con una llanura de inundación amplia $(5-15 \mathrm{~km})$ y costas bajas compuestas por albardones donde se depositan sedimentos con selvas en galería, bosques de sauces y alisos de río, palmares de Copenicia, bosques xerófilos chaqueños, sabanas y diversos humedales leníticos.

Obtuvimos 13,039 registros de 104 especies de serpientes (tabla 1) mediante la revisión de colecciones herpetológicas y de literatura confiable con localidad precisa (ver Arzamendia y Giraudo, 2009 para detalles), y la realización de 361 campañas de muestreo entre 1989 y 2013 , priorizando regiones poco conocidas y muestreadas con el objetivo de complementar los datos obtenidos en colecciones y literatura para minimizar posibles sesgos. Los mapas de distribución de los taxones se confeccionaron con Diva Gis 7.5 (Hijmans et al., 2005).

Con el objetivo de comparar afinidades y descubrir patrones biogeográficos, se usaron 2 métodos frecuentemente aplicadas en este tipo de comparaciones (López, Menni, Donato y Miquelarena, 2008; Luna-Cavazos, Romero-Mazanares y García-Moya, 2008): un análisis de parsimonia de endemismos 
(PAE, por sus siglas en inglés) y un análisis multivariado de agrupamiento (AMA). Para ambos se construyó una matriz de presencia (1) y ausencia (0) de especies (Anexo 1), donde las columnas representaron las taxones (caracteres) y las filas las áreas comparadas (tramos de los ríos y áreas fitogeográficas). En el PAE se agregó un área hipotética que no contiene especies para enraizar los árboles (Cué-Bär, Villaseñor, Morrone e Ibarra-Manríquez, 2006). Estos métodos ya fueron utilizados en biogeografía de fitoplancton, zooplancton, helmintos y plantas acuáticas de sistemas hidrográficos de Chile y México (Aguilar-Aguilar, Contreras-Medina, Martínez-Aquino, Salgado-Maldonado y González-Zamora, 2005; Mora-Olivo, Villaseñor, Luna-Vega y Morrone, 2008; Oyanedel, Vega-Retter, Scott, Hinojosa y Ramos-Jiliberto, 2008) y de peces para las cuencas argentinas (López et al., 2008). Ambos análisis fueron realizados mediante la comparación de afinidades entre los tramos de los ríos y entre los tramos de los ríos y las provincias fitogeográficas que los rodean (Paranaense, Chaqueña, del Espinal y Pampeana; fig. 1). Si un taxón presentó registros exclusivamente asociados a los grandes ríos en un área fitogeográfica determinada (por ejemplo, Hydrodynastes gigas exclusivamente en el río Paraná dentro de la provincia Pampeana) no fue considerado como presente en tal área fitogeográfica.

El PAE es un método que permite encontrar relaciones entre áreas basado en los patrones naturales de distribución en concordancia con sus taxones compartidos por medio de algoritmos de parsimonia, que dan como resultado una clasificación jerárquica de unidades biogeográficas (Crisci, Katinas y Posadas, 2000; López et al., 2008). La matriz (Anexo 1) se analizó con el programa TNT 1.1. (Goloboff, Farris y Nixon, 2008). Se aplicó una búsqueda heurística con 100 réplicas y permutación entre ramas con el algoritmo TBR. El soporte de las ramas fue basado en un análisis de Jacknife. A fin de caracterizar las áreas se utilizaron las especies exclusivas (sinapomorfías) para cada área, que equivalen a un clado (López et al., 2008).

El AMA fue usado para la clasificación de biotas (López et al., 2008), aunque las áreas son agrupadas mediante algoritmos matemáticos que muestran las distancias relativas entre grupos en relación con su composición taxonómica y consideran los taxones compartidos, así como ausencias compartidas y especies únicas. A diferencia del PAE que se basa exclusivamente en especies compartidas (López et al., 2008; Rosen, 1988, ver discusión en Escalante, 2009). Para construir la matriz de similitud se aplicó el coeficiente de Jaccard, comúnmente utilizado en comparaciones faunísticas (Van Jaarsveld et al., 1998) y se construyó el dendrograma mediante el método de agrupamiento promedio no ponderado (UPGMA). Se obtuvo el coeficiente cofenético para evaluar el grado de distorsión del análisis. Los análisis de conglomerados fueron realizados mediante el programa PAST (Hammer, Harter y Ryan, 2001).

\section{Resultados}

\section{Análisis de parsimonia de endemismos}

Se obtuvo un solo árbol (fig. 2) con un índice de consistencia de 0.578 y un índice de retención de 0.581. Los valores de

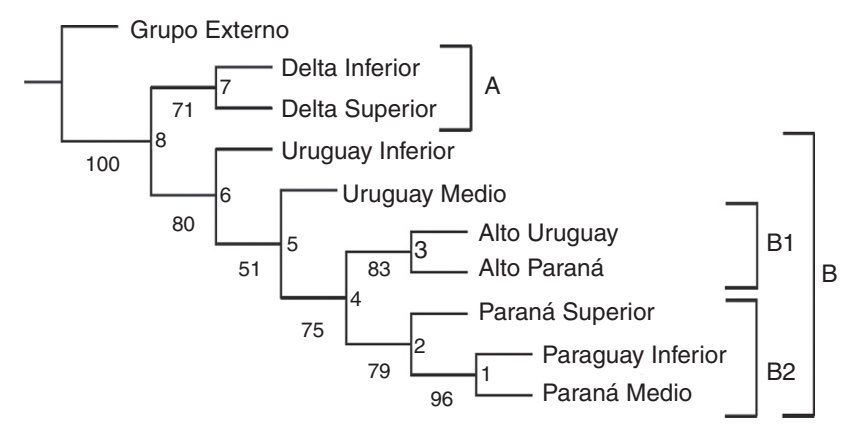

Figura 2. Cladograma obtenido con PAE comparando tramos de los grandes ríos de la cuenca del Plata. Valores de Jacknife se muestran debajo en las ramas y en los nodos se indican los números usados en en la tabla 2.

Jacknife fueron altos con todas las ramas sustentadas por más del $50 \%$. Se observan 2 grandes clados (fig. 2). El clado A formado por los tramos DS e Inferior (Jacknife del 71\%) queda definido por Amerotyphlops brongersmianus, Xenodon semicinctus, Phalotris bilineatus y Psomophis obtusus. El DI cuenta con una especie, Epictia munoai, no registrada en el DS, y en este tramo se encuentran especies como Helicops leopardinus, Hydrodynastes gigas y Leptophis ahaetulla marginatus, no registradas en el DI. El clado B (fig. 2) está formado por el Uruguay Inferior y Medio como basales y 2 subclados B1 y B2. El subclado B1 está conformado por el Alto Uruguay y Alto Paraná, comparten las siguientes especies (sinapomorfías): Liotyphlops beui, Atractus taeniatus, Erythrolamprus miliaris orinus, Spilotes pullatus, Tomodon dorsatus, Xenodon histricus, X. neuwiedii, Bothrops jararaca y Bothrops jararacussu. El subclado B2 está bien sustentado y conformado por: [(Paraguay Inferior-Paraná Medio) Paraná Superior]. Sus especies sinapomórficas son Mastigodryas bifossatus triseriatus, Clelia clelia, Hydrops caesurus, Leptodeira annulata pulchriceps, Lygophis meridionalis, Erythrolamprus poecilogyrus caesius, Mussurana bicolor, Philodryas olfersii latirostris, Bothrops diporus; y las especies exclusivas del Paraguay Inferior-Paraná Medio son Atractus snethlageae, Lygophis dilepis, Philodryas mattogrossensis, Thamnodynastes chaquensis, Xenodon pulcher y Micrurus pyrrhocryptus (tabla 2).

Tabla 2

Lista de sinapomorfías exclusivas que sustentan los nodos del cladograma comparando tramos de los ríos.

\begin{tabular}{ll}
\hline Nodos & Sinapomorfías \\
\hline Nodo 1 (PI-PM) & $22,50,63,79,89,95$ \\
Nodo 2 (PS (PI-PM)) & $4,14,25,27,37,46,48,52$, \\
& 64,103 \\
Nodo 3 (AU-AP) & $2,23,36,15,84,86,87,99$, \\
& 100 \\
Nodo 4 ((AU-AP) (PS (PI-PM))) & 4 \\
Nodo 5 (UM ((AU-AP) (PS (PI-PM))) & $3,12,75,87$ \\
Nodo 6 (UI (UM ((AU-AP) (PS (PI-PM))) & $9,24,51,61,92,104$ \\
Nodo 7 (DI-DS) & $57,72,90$
\end{tabular}

Los números corresponden a la lista de especies de la tabla 1.

Tramos de los ríos: AP: Alto Paraná; PS: Paraná Superior; PM: Paraná Medio; DS: Delta Superior; DI: Delta Inferior; AU: Alto Uruguay; UM: Uruguay Medio; UI: Uruguay Inferior; PI: Paraguay Inferior.

Regiones fitogeográficas: PAR: Paranaense; CHH: Chaco Húmedo; ESP: Espinal; PAM: Pampeana. 


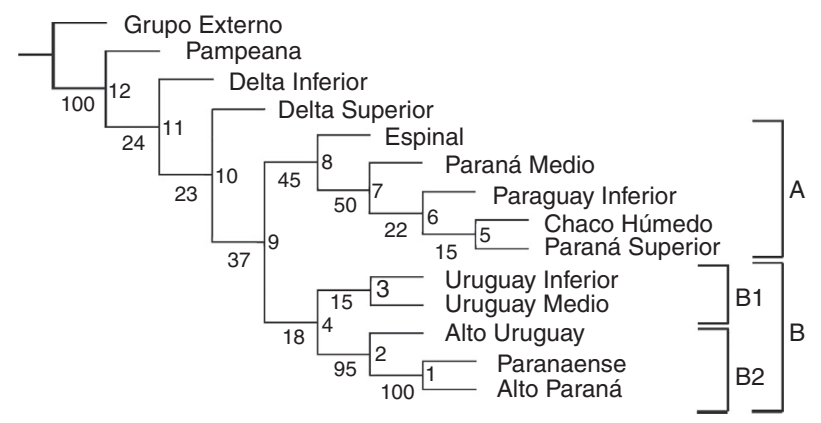

Figura 3. Cladograma obtenido con PAE comparando los tramos de los grandes ríos de la cuenca del Plata y las regiones fitogeográficas. Valores de Jacknife se muestran debajo de las ramas y en los nodos se indican los números usados en la tabla 3 .

El árbol obtenido incluyendo los tramos de los ríos y las regiones fitogeográficas (fig. 3) muestra bajos valores de Jacknife en la mayoría de los nodos con soportes menores a $50 \%$. El índice de consistencia es 0.500 y el índice de retención es 0.652. Se distingue el clado A, formado por [(((Paraná SuperiorChaco Húmedo) Paraguay Inferior) Paraná Medio) Espinal], con soporte del $45 \%$. El clado B queda definido por especies de la región Paranaense y exclusivas de los ríos en estos sectores, Chironius bicarinatus, Philodryas olfersii olfersii y Micrurus altirostris (fig. 3). Este clado comprende 2 subclados: B1, con bajo soporte (15\%), conformado por el Uruguay Medio e Inferior y definido por Phalotris lemniscatus y Taeniophalus poecilopogon; y B2, con soporte muy elevado (95\%), conformado por [(Alto Paraná-Paranaense) Alto Uruguay] que presentan 13 sinapomorfías (tabla 3).

Tabla 3

Lista de sinapomorfías exclusivas que sustentan los nodos del cladograma comparando tramos de los ríos y las regiones fitogeográficas.

\begin{tabular}{|c|c|}
\hline Nodos & Sinapomorfías \\
\hline Nodo 1 (AP-PAR) & $\begin{array}{l}3,7,10,26,30,40,47,55 \\
73,74,91,101\end{array}$ \\
\hline Nodo 2 (AU (AP-PAR)) & $\begin{array}{l}2,13,15,19,23,36,38,76, \\
83,86,88,100,102\end{array}$ \\
\hline Nodo 3 (UI-UM) & 58,78 \\
\hline Nodo 4 ((UI-UM)(AU (AP-PAR))) & $9,65,92$ \\
\hline Nodo 5 (CHH-PS) & 67 \\
\hline Nodo 6 (PI (CHH-PS)) & - \\
\hline Nodo 7 (PM (PI (CHH-PS))) & $\begin{array}{l}8,14,25,27,33,44,46,52 \\
63,64,77,79\end{array}$ \\
\hline Nodo 8 (ESP (PM (PI (CHH-PS)))) & $\begin{array}{l}4,11,37,48,50,68,89,93 \\
95,103\end{array}$ \\
\hline $\begin{array}{l}\text { Nodo } 9((\text { ESP }(\text { PM }(\text { PI }(\text { CHH-PS })))) \\
\quad((\text { UI-UM })(\text { AU }(\text { AP-PAR }))))\end{array}$ & $24,75,87,104$ \\
\hline $\begin{array}{l}\text { Nodo } 10(\mathrm{DS} \text { (ESP (PM (PI } \\
\quad(\mathrm{CHH}-\mathrm{PS}))))((\mathrm{UI}-\mathrm{UM})(\mathrm{AU}(\mathrm{AP}-\mathrm{PAR}))))\end{array}$ & 12 \\
\hline $\begin{array}{l}\text { Nodo } 11 \text { (DI (DS (ESP (PM (PI } \\
\quad(\text { CHH-PS })))((\text { UI-UM })(\text { AU }(\text { AP-PAR }))))\end{array}$ & $1,35,42,43,80,82$ \\
\hline
\end{tabular}

Los números corresponden a la lista de especies de la tabla 1.

Tramos de los ríos: AP: Alto Paraná; PS: Paraná Superior; PM: Paraná Medio; DS: Delta Superior; DI: Delta Inferior; AU: Alto Uruguay; UM: Uruguay Medio; UI: Uruguay Inferior; PI: Paraguay Inferior

Regiones fitogeográficas: PAR: Paranaense; CHH: Chaco Húmedo; ESP: Espinal; PAM: Pampeana.

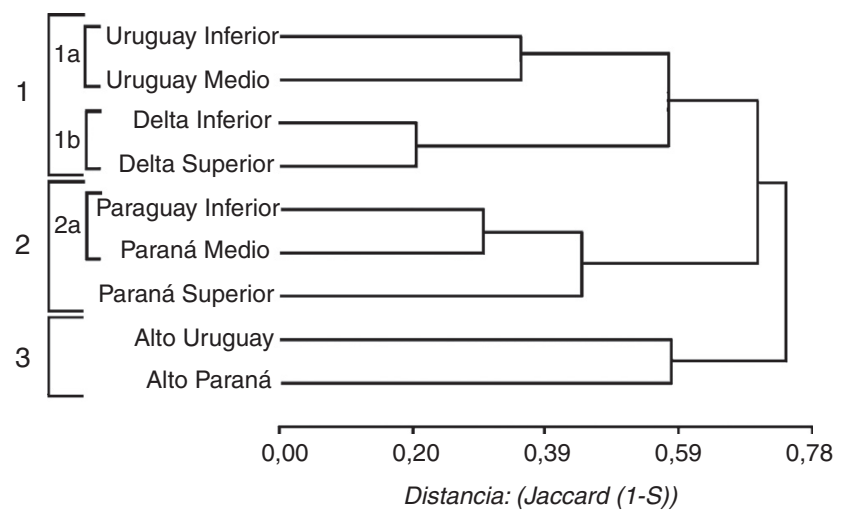

Figura 4. Dendrograma de similitud usando el coeficiente de Jaccard y el método UPGMA (correlación cofenética $=0.864$ ) comparando los diferentes tramos de los grandes ríos de la cuenca del Plata.

\section{Análisis multivariado}

Las relaciones de similitud faunística entre los tramos de los ríos se indican en la figura 4. Se diferenciaron 3 grandes grupos: el primero incluye 2 subgrupos, el núcleo de mayor similitud está formado por el DI (20 especies) y Superior (21), que comparten 18 taxones, y se relaciona con el grupo constituido por el Uruguay Medio (34) e Inferior (22) que comparten 22 especies entre ellos (tabla 4). El segundo grupo está conformado por un subgrupo de mayor similitud constituido por el Paraná Medio (51 especies) y el Paraguay Inferior (50), que comparten 42 de las especies en cada tramo (tabla 4). Este grupo se relaciona con el Paraná Superior (57 especies), con el cual comparten especies asociadas a los ríos como Hydrops caesurus, Clelia clelia y Lygophis meridionalis. El tercer grupo contiene al Alto Paraná (48 especies) y al Alto Uruguay (46), este núcleo comparte 29 taxones, aunque no poseen especies exclusivas de los ríos (tabla 4).

Los análisis de similitud entre los tramos de los ríos considerados y las regiones fitogeográficas dieron como resultado 3 grandes grupos indicados como 1, 2 y 3 en la figura 5. El grupo 1 incluye el núcleo de mayor similitud formado por el DI y

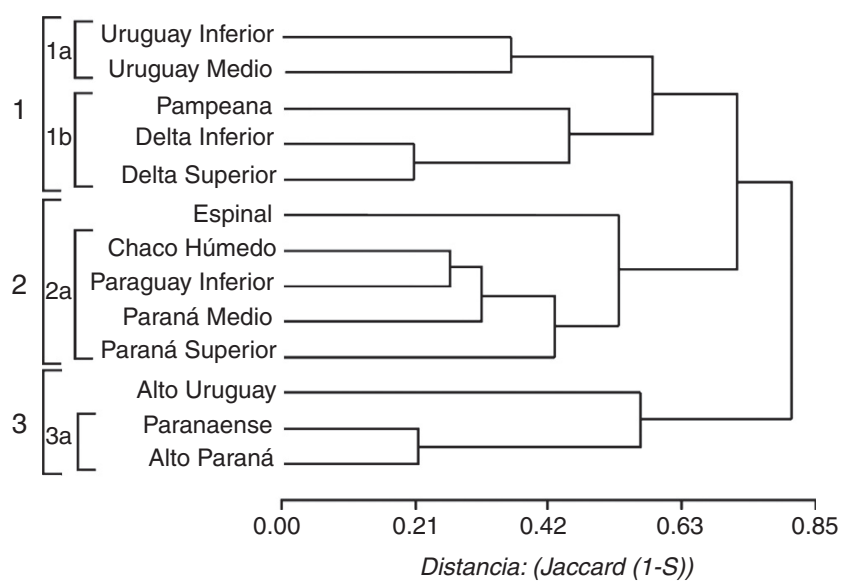

Figura 5. Dendrograma de similitud usando el coeficiente de Jaccard y el método UPGMA (correlación cofenética $=0.874$ ) comparando los tramos de los grandes ríos y las regiones fitogeográficas. 
Tabla 4

Matriz indicando la riqueza de taxones compartidos por los tramos de los ríos en las regiones fitogeográficas (en negrita en diagonal superior derecha) y distancia a partir del complemento del índice de similitud de Jaccard (1-S) (parte inferior-izquierda de la diagonal).

\begin{tabular}{llllllllllllll}
\hline & AP & PS & PM & DS & DI & AU & UM & UI & PI & PAR & CHH & ESP & PAM \\
\hline AP & $\mathbf{4 8}$ & $\mathbf{2 7}$ & $\mathbf{1 4}$ & $\mathbf{5}$ & $\mathbf{5}$ & $\mathbf{2 9}$ & $\mathbf{1 2}$ & $\mathbf{7}$ & $\mathbf{1 1}$ & $\mathbf{4 2}$ & $\mathbf{1 4}$ & $\mathbf{7}$ & $\mathbf{2}$ \\
PS & 0.65 & $\mathbf{5 7}$ & $\mathbf{3 8}$ & $\mathbf{1 5}$ & $\mathbf{1 4}$ & $\mathbf{3 3}$ & $\mathbf{2 5}$ & $\mathbf{1 6}$ & $\mathbf{3 6}$ & $\mathbf{2 4}$ & $\mathbf{3 9}$ & $\mathbf{2 1}$ & $\mathbf{8}$ \\
PM & 0.84 & 0.46 & $\mathbf{5 1}$ & $\mathbf{1 9}$ & $\mathbf{1 6}$ & $\mathbf{2 2}$ & $\mathbf{2 4}$ & $\mathbf{1 6}$ & $\mathbf{4 2}$ & $\mathbf{1 1}$ & $\mathbf{3 9}$ & $\mathbf{2 8}$ & $\mathbf{1 2}$ \\
DS & 0.92 & 0.76 & 0.64 & $\mathbf{2 1}$ & $\mathbf{1 8}$ & $\mathbf{1 5}$ & $\mathbf{1 7}$ & $\mathbf{1 3}$ & $\mathbf{1 4}$ & $\mathbf{4}$ & $\mathbf{1 4}$ & $\mathbf{1 3}$ & $\mathbf{1 2}$ \\
DI & 0.92 & 0.78 & 0.71 & 0.22 & $\mathbf{2 0}$ & $\mathbf{1 5}$ & $\mathbf{1 7}$ & $\mathbf{1 4}$ & $\mathbf{1 2}$ & $\mathbf{5}$ & $\mathbf{1 2}$ & $\mathbf{1 2}$ & $\mathbf{1 3}$ \\
AU & 0.55 & 0.53 & 0.71 & 0.71 & 0.71 & $\mathbf{4 6}$ & $\mathbf{2 1}$ & $\mathbf{1 6}$ & $\mathbf{1 9}$ & $\mathbf{2 8}$ & $\mathbf{2 2}$ & $\mathbf{1 4}$ & $\mathbf{9}$ \\
UM & 0.83 & 0.62 & 0.61 & 0.55 & 0.54 & 0.64 & $\mathbf{3 4}$ & $\mathbf{2 2}$ & $\mathbf{1 8}$ & $\mathbf{1 1}$ & $\mathbf{2 0}$ & $\mathbf{1 8}$ & $\mathbf{1 4}$ \\
UI & 0.89 & 0.75 & 0.72 & 0.57 & 0.50 & 0.69 & 0.35 & $\mathbf{2 2}$ & $\mathbf{1 0}$ & $\mathbf{8}$ & $\mathbf{1 1}$ & $\mathbf{1 3}$ & $\mathbf{1 1}$ \\
PI & 0.87 & 0.49 & 0.29 & 0.75 & 0.79 & 0.75 & 0.73 & 0.84 & $\mathbf{5 0}$ & $\mathbf{9}$ & $\mathbf{4 2}$ & $\mathbf{2 4}$ & $\mathbf{8}$ \\
PAR & 0.22 & 0.70 & 0.88 & 0.94 & 0.92 & 0.58 & 0.85 & 0.87 & 0.90 & $\mathbf{4 8}$ & $\mathbf{1 2}$ & $\mathbf{6}$ & $\mathbf{2}$ \\
CHH & 0.84 & 0.44 & 0.39 & 0.76 & 0.80 & 0.71 & 0.70 & 0.83 & 0.30 & 0.86 & $\mathbf{5 2}$ & $\mathbf{2 5}$ & $\mathbf{9}$ \\
ESP & 0.90 & 0.68 & 0.47 & 0.66 & 0.68 & 0.77 & 0.61 & 0.67 & 0.57 & 0.92 & 0.56 & $\mathbf{3 0}$ & $\mathbf{1 3}$ \\
PAM & 0.97 & 0.88 & 0.78 & 0.52 & 0.43 & 0.83 & 0.61 & 0.59 & 0.86 & 0.97 & 0.85 & 0.61 & $\mathbf{1 6}$ \\
\hline
\end{tabular}

La diagonal indica la riqueza de taxones en cada tramo.

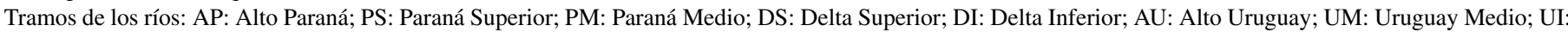
Uruguay Inferior; PI: Paraguay Inferior.

Regiones fitogeográficas: PAR: Paranaense; CHH: Chaco Húmedo; ESP: Espinal; PAM: Pampeana.

Superior (tabla 4) que se une con la provincia fitogeográfica Pampeana y con menor similitud con el núcleo constituido por el Uruguay Medio y el Inferior. El grupo 2 incluye al Paraguay Inferior con el Chaco Húmedo, que comparten 42 especies. Este núcleo se relaciona con el Paraná Medio y luego con el Paraná Superior, y se une con menor similitud con la provincia del Espinal (fig. 5). El grupo 3 está constituido por la provincia Paranaense y Alto Paraná, núcleo de mayor similitud (complemento de Jaccard 0.22) que comparten 43 especies de las 48 que contiene el Alto Paraná, este nodo se relaciona con el Alto Uruguay, con el cual comparte 25 taxones.

\section{Discusión}

Los resultados obtenidos brindaron evidencias para discutir las hipótesis sobre las relaciones entre los tramos de los ríos planteadas en la introducción.

\section{Hipótesis 1}

El Alto Paraná presenta mayor afinidad con el Alto Uruguay respecto a otros tramos del Paraná. Principalmente los resultados del PAE y también los de AMA apoyan fuertemente esta hipótesis, formando un clado con elevado soporte y varias especies endémicas que relacionan biogeográficamente al Alto Paraná con mayor afinidad faunística con el Alto Uruguay respecto a otros tramos del Paraná. Esto coincide con lo expresado por varios autores que estudiaron organismos acuáticos y terrestres y encontraron concordancias entre las faunas de ambos tramos (Bonetto, 1961; Bonetto y Drago, 1968; Giraudo, 2001; López et al., 2008; Ringuelet, 1961). Las nacientes y principales afluentes de los ríos Paraná y Uruguay se ubican y fluyen principalmente en las Serras do Mar y Geral, en la ecorregión de la selva Atlántica-Paranaense (Arzamendia y Giraudo, 2009; Bonetto, 1986; Di Persia y Neiff, 1986; Paoli et al., 2000), y a pesar de que el Alto Paraná se conecta a través del Paraná
Superior con el eje Paraguay-Paraná Medio, la mayoría de las especies atlántico-paranaenses no habitan en el Paraná Medio, por lo que las conexiones geográficas de estos ríos no pueden explicar exclusivamente estos patrones. Desde una perspectiva histórica-geomorfológica, luego de la última ingresión marina terciaria, los ríos de la cuenca del Plata se desplazaron repetidas veces (Castellanos, 1965; Orfeo, 2005; Paoli et al., 2000). El Alto Paraná estuvo conectado durante largos periodos con el Alto Uruguay y, por lo tanto, históricamente aislado del Paraná Medio (ver Arzamendia y Giraudo, 2009 para más detalles). Adicionalmente, el Alto Paraná y el Alto Uruguay presentan condiciones ecológicas similares constituidas por sus cauces estrechos, con lechos basálticos y numerosos rápidos con costas en barrancas elevadas que contienen selva Paranaense-Atlántica, con especies de serpientes características de esta diversa ecorregión (Arzamendia y Giraudo, 2009). La influencia faunística de la provincia Paranaense en los tramos Alto Paraná y Alto Uruguay es apoyada fuertemente por los resultados de PAE y AMA, que muestran un clado con alto soporte y un agrupamiento con elevado coeficiente de Jaccard, en coincidencia con los aportes zoogeográficos de Bonetto (1961) y Ringuelet (1961), quienes sostuvieron que la fauna del Alto Paraná y Alto Uruguay están dominados por elementos paranaenses.

\section{Hipótesis 2}

El Paraná Medio tiene mayor afinidad con el Paraguay Inferior respecto de otros tramos del propio río Paraná. Esta hipótesis se ve apoyada por la formación de un grupo con elevada similitud entre los ensambles de serpientes y de un clado con elevado soporte con especies sinapomórficas en el PAE, que relaciona estrechamente al Paraguay Inferior con el Paraná Medio con respecto a los demás tramos del río. Se une a este grupo/clado basalmente el tramo del Paraná Superior, aunque con menor soporte y similitud de Jaccard. Esta hipótesis concuerda con lo indicado para otros grupos taxonómicos 
(moluscos, rotíferos, crustáceos, peces y macrófitas), por autores que sostuvieron que existe continuidad faunística entre el eje potámico Paraguay-Paraná Medio (Bonetto, 1961; Bonetto y Drago, 1968; José de Paggi, 1990; López et al., 2008; Morrone y Lopretto, 2001; Oakley et al., 2005; Ringuelet, 1961, 1975). Tal afinidad faunística puede deberse a la similitud y continuidad de ambientes y características ecológicas de los tramos considerados, con amplias llanuras de inundación, abundantes humedales leníticos, vegetación acuática y palustre similar y selva marginal bien desarrollada en el río Paraguay que se extiende hasta el sector norte del Paraná Medio (Menalled y Adámoli, 1995; Oakley et al., 2005), características que se extienden en el Paraná Superior, sector aguas arriba del Paraná Medio.

\section{Hipótesis 3}

El DS tiene mayor afinidad con el Paraná Medio, mientras que el DI tiene mayor afinidad con el río Uruguay Inferior. Los resultados obtenidos no apoyan la hipótesis formulada por Menalled y Adámoli (1995) para árboles y arbustos. El DS no aparece en los análisis relacionados con el Paraná Medio ni el DI con el río Uruguay Inferior. En los análisis de similitud los Deltas del Paraná forman un núcleo, con elevado coeficiente de Jaccard, que está relacionado con el grupo del Uruguay Medio e Inferior (fig. 4). Este patrón es coincidente con la afirmación de Menalled y Adámoli (1995), quienes le atribuyeron al río Uruguay ser la principal ruta de migración de elementos tropicales que conforman la comunidad del Bajo Delta en latitudes templadas, a pesar de que el Paraná ha tenido una mayor preponderancia en la formación geomorfológica del Delta. En el análisis de endemicidad (PAE), los Delta también forman un subgrupo que es basal y está separado del resto de los tramos de los ríos, y a diferencia del análisis de similitud, el Uruguay Inferior y Uruguay Medio son basales a 2 clados conformados con alto soporte: (Alto Paraná-Alto Uruguay)-[(Paraná MedioParaguay) Paraná Superior], debido a que comparten taxones tropicales (e.g., Chironius bicarinatus) que no alcanzan en su distribución meridional la elevada latitud donde se encuentran los Deltas. Las especies que se muestran como sinapomorfías del clado conformado por ambos Deltas son especies pampeanas como Xenodon semicinctus y Psomophis obtusus. A diferencia que lo que ocurre con lo indicado para árboles y arbustos (Menalled y Adámoli, 1995), la comunidad de serpientes de los Deltas se encuentra simplificada con pérdida de especies respecto a tramos superiores, lo que probablemente se debe al aumento de la latitud que se correlaciona con una disminución de la temperatura (Giraudo, Arzamendia y López, 2007), que podría afectar en mayor grado a los reptiles heterotermos respecto a las plantas. La fauna de serpientes de ambos Deltas tiene una baja riqueza (DS con 21 taxones y DI con 20). Helicops leopardinus, Hydrodynastes gigas y Leptophis ahaetulla marginatus son las únicas especies presentes en el DS que no fueron registradas en el DI. Estas especies se distribuyen a través del eje ParaguayParaná Medio y alcanza el DS, coincidiendo estos taxones con los patrones indicados por Menalled y Adámoli (1995) para vegetación. Epictia munoai y Philodryas olfersii olfersii alcanzan el DI y no fueron registradas en el DS. La primera tiene una amplia distribución, y la segunda se distribuye desde las selvas Paranaenses de Misiones y a través del río Uruguay alcanza el DI. Por ser las serpientes animales ectotermos, su distribución y aspectos de su historia de vida (reproducción, actividad, alimentación y metabolismo) se ven afectados fuertemente por la temperatura (Pough et al., 1998; Rey Benayas y de la Montaña, 2003), siendo esta una posible causa de la simplificación de los ensambles de serpientes en los Deltas. La ausencia de especies influye en la afinidad faunística entre los DI y DS con los tramos contiguos septentrionales.

En el análisis de similitud y el PAE se evidencia una fuerte relación entre los tramos de los ríos y la región fitogeográfica que estos atraviesan. El Alto Paraná forma un núcleo/clado en conjunto con la provincia biogeográfica Paranaense y con el Alto Uruguay, debido a que sus costas e islas presentan selva Paranaense. En ambos análisis, AMA y PAE, se define un grupo/clado que reúne a las áreas biogeográficas Chaco Húmedo y del Espinal (dominio Chaqueño) con el Paraná Superior, Medio y Paraguay Superior, donde el Espinal queda como basal en el PAE y con menor similitud en AMA, probablemente porque se caracteriza mayormente por la desaparición de especies chaqueñas, más que por contener elementos propios, razón por la cual el Espinal ha sido cuestionado o tratado como un distrito de la provincia Pampeana (e.g., Morrone, 2014). Los análisis de PAE permiten reconocer componentes bióticos y unidades biogeográficas caracterizadas por especies exclusivas, posibilitando discutir distintos criterios de regionalización biogeográfica de estos grandes ríos. Por ejemplo, Cabrera (1994) incluyó al río Paraná dentro de la provincia biogeográfica Paranaense, mientras que Dinerstein et al. (1995) consideró al río Paraná Medio-Paraguay Inferior como una ecorregión independiente (sabanas inundadas del Paraná), criterio en parte seguido por Burkart, Bárbaro, Sánchez y Gómez (1999), que denominaron a esta ecorregión como Delta e islas del Paraná. Nuestros análisis muestran una importante afinidad biogeográfica con el Chaco Húmedo, incluyendo también al Paraná Superior, y evidencian que el Paraná Medio, Paraguay Inferior y el Paraná Superior podrían incluirse como parte de esta última ecorregión; mientras que el Alto Paraná y los tramos del río Uruguay deben ser considerados dentro de la provincia biogeográfica Paranaense.

Los tramos más australes de los ríos DS y DI comparten elementos pampeanos, corroborado por su mayor similitud faunística con la región fitogeográfica Pampeana, y se relacionan con el Uruguay Inferior y Medio como se discutió anteriormente. Sin embargo, el cladograma del PAE muestra la influencia de especies tropicales paranaenses entre los tramos Uruguay Medio y Uruguay Inferior, que alcanzan mayores latitudes por el río Uruguay en coincidencia con lo indicado por Arzamendia y Giraudo (2009), Capllonch, Ortiz y Soria (2008), Giraudo y Arzamendia (2004) y Nores et al. (2005). Los grandes ríos del Plata generan un importante intercambio biótico con las regiones biogeográficas aledañas que atraviesan, representando áreas con elevada riqueza por el aporte de especies de serpientes tropicales de distintos linajes biogeográficos (e.g., paranaenses, amazónicos y del Pantanal, ver Arzamendia y Giraudo, 2009) en conjunción con fauna de regiones biogeográficas 
subtropicales-templadas (chaqueñas y pampeanas), y representan regionalmente áreas complejas (Morrone y Gutiérrez, 2005). Los patrones de distribución de las especies y relaciones faunísticas entre los tramos de los grandes ríos del Plata pueden ser explicados por la interacción de procesos históricos (eventos geomorfológicos pasados que causaron el desplazamiento de los grandes ríos y cambios en sus conexiones), principalmente en combinación con factores ecológicos (similitudes hidrológicas o ambientales) y geográficos (grado de conexión actual de los ríos y de regiones biogeográficas circundantes), debido a que el simple efecto de los ríos como vías de dispersión de especies, resultan insuficientes para explicar la mayoría de los patrones de distribución encontrados (Arzamendia y Giraudo, 2009).

Las afinidades y relaciones biogeográficas obtenidas aquí mediante el análisis de las serpientes con PAE y métodos multivariados, junto a los análisis biogeográficos previos que reportaron la existencia de nodos biogeográficos, en diferentes grupos de organismos (Arzamendia y Giraudo, 2009, 2012; Mayén-Estrada y Aguilar-Aguilar, 2012; Morrone y Lopretto, 1994, 2001), nos permiten proponer a los grandes ríos de la cuenca del Plata, como verdaderas áreas de transición biogeográfica, en el sentido que le dan Ferro y Morrone (2014). Estas constituyen áreas complejas en sus distintos tramos debido a la superposición y sustitución parcial de componentes bióticos (conjuntos de taxones que comparten una distribución geográfica similar producto de una historia evolutiva común) con diferentes historias y afinidades biogeográficas y evolutivas. Estas zonas de transición biogeográfica son lugares donde los diferentes linajes evolutivos coexisten y, por lo tanto, presentan una alta diversidad filogenética (Ferro y Morrone, 2014), por lo que constituyen áreas importantes para la conservación, particularmente cuando las transiciones biogeográficas, como ocurre con los grandes ríos de la cuenca del Plata, muestran una alta diversidad y un relativo buen estado de conservación respecto a tierras aledañas bajo uso productivo más intensivo (Arzamendia y Giraudo, 2009, 2012; Giraudo et al., 2007).

\section{Agradecimientos}

A quienes financiaron nuestro trabajo: CONICET (PIP 2011-0355), Universidad Nacional del Litoral (CAID- 2011524-UNL) y ANPCYT (PICT 2011-1526 y PICT-2013-2203 FONCYT). Agradecemos especialmente a M. ' . Eugenia Rodríguez, la familia Escalante, Raúl Abramson, Eduardo Etchepare, Claudia Szumik, Maximiliano Cristaldi, Juan Andrés Sarquis, a los curadores de los museos FML, MACN, UNNEC, MLP y CIES, así como al personal del INALI, quienes facilitaron nuestro trabajo.

\section{Anexo 1.}

Matriz de presencia/ausencia de 104 taxones de serpientes (nombres de las especies en orden numérico en el anexo en los tramos de los ríos [AP : Alto Paraná; PS : Paraná Superior; PM : Paraná Medio; DS : Delta Superior; DI : Delta Inferior; AU : Alto Uruguay; UM : Uruguay Medio; UI : Uruguay Inferior y PI:Paraguay Inferior]) y las regiones fitogeográficas [PAR : Paranaense; CHH:Chaco Húmedo; ESP:Espinal y PAM : Pampeana]).

\begin{tabular}{|c|c|c|c|c|c|c|c|c|c|c|c|c|c|c|c|c|c|c|c|c|c|}
\hline & 5 & 10 & 15 & 20 & 25 & 30 & 35 & 40 & 45 & 50 & 55 & 60 & 65 & 70 & 75 & 80 & 85 & 90 & 95 & 100 & 104 \\
\hline AP & 1 & & & 1 & & & & & 0 & 0 & & & & & & 00 & 0 & 11 & 10 & & 101 \\
\hline PS & 10111 & 00110 & 11110 & 1101 & 00110 & 11000 & 01110 & 11 & 111 & 01001 & 101 & 10101 & 01111 & 11000 & 00011 & 01001 & 01001 & 10 & & 11000 & 0111 \\
\hline PM & 10110 & 00100 & 11010 & 00000 & 11110 & 10100 & 01010 & 11010 & 0111 & 01110 & 10101 & 10001 & 11010 & 11000 & 10010 & 01011 & 01011 & 01010 & 01101 & 01000 & 0011 \\
\hline DS & 10000 & 00000 & 01000 & 00000 & 00000 & 00100 & 00010 & 10010 & 1110 & 00100 & 00001 & 10001 & 00010 & 000 & 10000 & 00001 & 01011 & 00100 & 00000 & 01000 & 0000 \\
\hline DI & 10001 & 00000 & 00000 & 00000 & 00000 & 00100 & 00010 & 10010 & 01000 & 00100 & 00001 & 10001 & 00110 & 00000 & 10000 & 00001 & 01011 & 00100 & 00000 & 01000 & 0000 \\
\hline $\mathrm{AU}$ & 11001 & 00110 & 01101 & 01101 & 01100 & 00100 & 00111 & 10100 & 01100 & 00101 & 00001 & 00001 & 00110 & 10000 & 00011 & 10000 & 01111 & 10011 & 01110 & 11011 & 0100 \\
\hline UM & 00100 & 00010 & 11001 & 00000 & 00100 & 00100 & 00010 & 10011 & 01000 & 00101 & 00001 & 01001 & 00111 & 01000 & 10010 & 01101 & 01011 & 00110 & 01000 & 01000 & 0001 \\
\hline UI & 00000 & 00010 & 00000 & 00000 & 00100 & 00100 & 00010 & 10010 & 01000 & 00101 & 00000 & 01001 & 00110 & 01000 & 00000 & 00101 & 01011 & 00000 & 01000 & 01000 & 0001 \\
\hline PI & 10011 & 10100 & 11011 & 00010 & 10110 & 10000 & 010 & 11 & & 11 & & 01 & & & & & 01 & & & 01000 & 0011 \\
\hline PAR & 11101 & 01011 & 00100 & 01101 & 01101 & 00111 & 00001 & 00101 & 10 & 10001 & 10 & 00100 & 00100 & 00 & 01 & 00 & 00 & 11 & 10 & 111 & 1101 \\
\hline $\mathrm{CHH}$ & 10011 & 00100 & 11011 & 11011 & 00100 & 10000 & 01010 & 11000 & 10110 & 01011 & 10011 & 10111 & 11011 & 11001 & 10010 & 01011 & 00001 & 01010 & 00111 & 01000 & 0011 \\
\hline ESP & 10010 & 00000 & 10000 & 00000 & 00100 & 00100 & 00000 & 01000 & 00010 & 01110 & 00001 & 11001 & 00010 & 11000 & 10010 & 00101 & 00011 & 01010 & 00101 & 01000 & 0011 \\
\hline PAM & 00001 & 00000 & 00000 & 00000 & 00000 & 00100 & 00000 & 00010 & 00000 & 00100 & 00001 & 11001 & 00010 & 01000 & 10000 & 00100 & 00011 & 00100 & 00000 & 01000 & 0000 \\
\hline
\end{tabular}




\section{Referencias}

Aguilar-Aguilar, R., Contreras-Medina, R., Martínez-Aquino, A., SalgadoMaldonado, G. y González-Zamora, A. (2005). Aplicación del análisis de parsimonia de endemismos (PAE) en los sistemas hidrológicos de México: un ejemplo con helmintos parásitos de peces dulceacuícolas. En J. Llorente y J. J. Morrone (Eds.), Regionalización biogeográfica en Iberoamérica y tópicos afines: primeras jornadas biogeográficas de la red iberoamericana de biogeografía y entomología sistemática (RIBES XII.I-CYTED) (pp. 227-239). México, D.F.: Las Prensas de Ciencias, Facultad de Ciencias, Universidad Nacional Autónoma de México.

Arzamendia, V. y Giraudo, A. R. (2009). Influence of large South American rivers of the Plata Basin on distributional patterns of tropical snakes: A panbiogeographical analysis. Journal of Biogeography, 36, 1739-1749.

Arzamendia, V. y Giraudo, A. R. (2012). A panbiogeographical model to prioritize areas for conservation along large rivers. Diversity and Distributions, $18,168-179$.

Bonetto, A. A. (1961). Acerca de la distribución geográfica de las náyades en la República Argentina. Physis, 22, 259-268.

Bonetto, A. A. (1986). The Paraná River system. En B. R. Davis y K. F. Walker (Eds.), The ecology of river systems (pp. 541-555). Dordrecht, Holanda: Dr. W. Junk Publishers.

Burkart, R., Bárbaro, N., Sánchez, R.O. y Gómez, D.A. (1999). Eco-regiones de la Argentina. Buenos Aires: Programa de Desarrollo Institucional, Componente de Política Ambiental, Administración de Parques Nacionales.

Bonetto, A. A. y Drago, E. (1968). Consideraciones faunísticas en torno a la delimitación de los tramos superiores del río Paraná. Physis, 27, $427-444$.

Cabrera, A. L. (1994). Regiones fitogeográficas argentinas. Enciclopedia Argentina de Agricultura y Jardinería, 2, 1-85.

Cabrera, A. L. y Willink, A. (1980). Biogeografía da América Latina. (Segunda edición). Washington, D.C: Secretaria General de Organización de los Estados Americanos.

Capllonch, P., Ortiz, D. y Soria, K. (2008). Importancia del litoral fluvial argentino como corredor migratorio de aves. Instituto Superior de Correlación Geológica, Miscelánea, 17, 107-120.

Castellanos, A. (1965). Estudio fisiográfico de la provincia de Corrientes. Pub. 49. Rosario: Instituto Fisiografía y Geología.

Cei, J. M. (1993). Reptiles del noroeste, nordeste y este de la Argentina. Herpetofauna de las selvas subtropicales, Puna y Pampas. Monografía. 14. Torino: Museo Regionale di Scienze Naturali Torino.

Crisci, J. V., Katinas, L. y Posadas, P. (2000). Introducción a la teoría y práctica de la biogeografía histórica. Buenos Aires: Sociedad Argentina de Botánica.

Cué-Bär, E. M., Villaseñor, J. L., Morrone, J. J. y Ibarra-Manríquez, G. (2006). Identifying priority areas for conservation in mexican tropical deciduos forest based on tree species. Interciencia, 31, 712-719.

Di Persia, D. H. y Neiff, J. J. (1986). The Uruguay River system. En B. R. Davis y K. F. Walker (Eds.), The ecology of river systems (pp. 599-621). Dordrecht, The Netherlands: Dr. W. Junk Publishers.

Dinerstein, E., Olson, D. M., Graham, D. J., Webster, A. L., Primm, S. A., Bookbinder, M. P., et al. (1995). A conservation assessment of the terrestrial ecoregions of Latin America and the Caribbean. Washington, D.C: WWFWorld Bank.

Escalante, T. (2009). Un ensayo sobre regionalización biogeográfica. Revista Mexicana de Biodiversidad, 80, 551-560

Ferro, I. y Morrone, J. J. (2014). Biogeographical transition zones: a search for conceptual synthesis. Biological Journal of the Linnean Society, 113, 1-12.

Galindo-Leal, C. y Câmara, I. G. (2003). Atlantic forest hotspot status: An overview. En C. Galindo-Leal e y I. G. Câmara (Eds.), Atlantic forest of the South America. Biodiversity status, threats, and outlook (pp. 3-23). Washington, D.C: Island Press.

Gallardo, J. M. (1979). Composición, distribución y origen de la herpetofauna chaqueña. En W. E. Duellman (Ed.), The South American herpetofauna: its origin, evolution, and dispersal (pp. 299-307). Museum of Natural history, The University of Kansas. Monograph 7.

Giraudo, A. R. (2001). La diversidad de serpientes de la selva Paranaense y del Chaco Húmedo: taxonomía biogeografía y conservación. Buenos Aires: Editorial LOLA.
Giraudo, A. R. y Arzamendia, V. (2004). ¿Son las planicies fluviales de la cuenca del Plata, corredores de biodiversidad? Los vertebrados amniotas como ejemplo. En J. J. Neiff (Ed.), Humedales de Iberoamérica (pp. 157-170). La Habana: CYTED, Programa Iberoamericano de Ciencia y Tecnología para el Desarrollo.

Giraudo, A. R., Arzamendia, V. y López, S. (2007). Reptiles. En M. Iriondo, J. C. Paggi, y J. Parma (Eds.), The middle Paraná River. Limnology of a subtropical Wetland (pp. 1341-1362). Heidelberg: Springer-Verlag Berlin.

Goloboff, P., Farris, J. y Nixon, K. (2008). TNT, a free program for phylogenetic analysis. Cladistics, 24, 774-786

Gudynas, E., Williams, J. D. y Azpelicueta, M. (1988). Morphology, ecology and biogeography of the South American caecilian Chthonerpeton indistinctum (Amphibia: Gymnophiona: Typhlonectidae). Zoologische Mededelingen, 62, 5-28.

Halffter, G. (1978). Un nuevo patrón de dispersión en la zona de transición Mexicana: el Mesoamericano de Montaña. Folia Entomológica Mexicana, 39-40, 219-222.

Hammer, O., Harter, D. A. T. y Ryan, P. D. (2001). Past: paleontological statistic software package for education and data analysis. Paleontología Electrónica, 4, 1-9 Recuperado el 23 septiembre 2014 de http://palaeoelectronica.org/2001_1/past/issue1_01.htm

Hijmans, R. J., Guarino, L., Jarvis, A., Brien, R. O., Mathur, P., Bussink, C. et al. (2005). DIVA-GIS. Ver. 5.2. Manual. Recuperado el 12 septiembre 2014 de http://www.diva-gis.org/

José de Paggi, S. (1990). Ecological and biogeographical remarks on the rotifer fauna of Argentina. Revista de Hidrobiología Tropical, 23, 297-311.

Kandus, P., Malvárez, I. y Madanes, N. (2003). Estudio de las comunidades de plantas herbáceas de las islas bonaerenses del Bajo Delta del río Paraná (Argentina). Darwiniana, 41, 1-16.

Lewis, J. P. y Franceschi, E. A. (1979). Notas sobre la dinámica de la vegetación del valle del río Paraná. Ecosur, 6, 145-163.

López, H. L., Menni, R. C., Donato, M. y Miquelarena, A. M. (2008). Biogeographical revision of Argentina (Andean and Neotropical Regions): An analysis using freshwater fishes. Journal of Biogeography, 35, $1564-1579$

Luna-Cavazos, M., Romero-Mazanares, A. y García-Moya, E. (2008). Afinidades en la flora genérica de piñonares del norte y centro de México: un análisis fenético. Revista Mexicana de Biodiversidad, 79, 449-458.

Malvárez, M. I. (1999). El delta del río Paraná como mosaico de humedales. En M. I. Malvárez (Ed.), Tópicos sobre humedales subtropicales y templados de América del Sur (pp. 35-53). Montevideo: Oficina Regional de Ciencia y Tecnológica de la UNESCO para América Latina.

Matteucci, S., Morello, J., Rodríguez, A. y Mendoza, N. (2004). El alto Paraná encajonado argentino-paraguayo: mosaicos de paisaje y conservación regional. Buenos Aires: Editorial de la Facultad de Arquitectura, Diseño y Urbanismo. Universidad de Buenos Aires.

Mayén-Estrada, R. y Aguilar-Aguilar, R. (2012). Track analysis and geographic distribution of some Lagenophrys Stein 1852 (Protozoa: Ciliophora: Peritrichia) species. Journal of Natural History, 46, 249-263.

Menalled, F. D. y Adámoli, J. M. (1995). A quantitative phytogeographic analysis of species richness in forest communities of the Paraná River Delta, Argentina. Vegetatio, 120, 81-90.

Mora-Olivo, A., Villaseñor, J. L., Luna-Vega, I. y Morrone, J. J. (2008). Patrones de distribución de la flora vascular acuática estricta en el estado de Tamaulipas, México. Revista Mexicana de Biodiversidad, 79, 435-448.

Morrone, J. J. (2004). La zona de transición sudamericana: caracterización y relevancia evolutiva. Acta Entomológica Chilena, 28, 41-50.

Morrone, J. J. (2014). Biogeographical regionalisation of the Neotropical region. Zootaxa, 3782, 1-110.

Morrone, J. J. y Gutiérrez, A. (2005). Do fleas (Insecta: Siphonaptera) parallel their mammal host diversification in the Mexican Transition Zone? Journal of Biogeography, 32, 1315-1325.

Morrone, J. J. y Lopretto, E. C. (1994). Distributional patterns of freshwater Decapoda (Crustacea: Malacostraca) in Southern South America: A panbiogeographic approach. Journal of Biogeography, 21, 97-109.

Morrone, J. J. y Lopretto, E. C. (2001). Trichodactylid biogeographic patterns (Crustacea: Decapoda) and the Neotropical region. Neotrópica, 47, 49-55. 
Nores, M., Cerana, M. M. y Serra, D. A. (2005). Dispersal of forest birds and trees along the Uruguay River in Southern South America. Diversity and Distributions, 11, 205-217.

Oakley, L. J., Prado, D. y Adámoli, J. (2005). Aspectos biogeográficos del corredor fluvial Paraguay-Paraná. Instituto Superior de Correlación Geológica,Miscelánea, 14, 245-258.

Orfeo, O. (2005). Historia geológica del Iberá, provincia de Corrientes, como escenario de biodiversidad. Instituto Superior de Correlación Geológica, Miscelánea, 14, 71-78.

Oyanedel, J. P., Vega-Retter, C., Scott, S., Hinojosa, L. F. y Ramos-Jiliberto, R. (2008). Finding patterns of distribution for freshwater phytoplankton, zooplankton and fish, by means of parsimony analysis of endemicity. Revista Chilena de Historia Natural, 81, 184-203.

Paoli, C., Iriondo, M. y García, N. (2000). Características de las cuencas de aporte. En C. Paoli y M. Schreider (Eds.), El río Paraná en su tramo medio. Contribución al conocimiento y prácticas ingenieriles en un gran río de llanura (pp. 27-68). Santa Fe: Centro de Publicaciones, Secretaría de Extensión, Universidad Nacional del Litoral.
Pough, F. H., Andrews, R., Cadle, J. E., Crump, M. L., Savitzky, A. H. y Wells, K. D. (1998). Herpetology. Nueva Jersey: Prentice Hall Upper Saddle River.

Rabinovich, J. E. y Rapoport, E. H. (1975). Geographical variation of diversity in Argentina passerine birds. Journal of Biogeography, 2, 141-157.

Rey Benayas, J. M. y de la Montaña, E. (2003). Identifying areas of high-value vertebrate diversity for strengthening conservation. Biological Conservation, 114, 357-370.

Ringuelet, R. A. (1961). Rasgos fundamentales de la zoogeografía de la Argentina. Physis, 22, 151-170.

Ringuelet, R. A. (1975). Zoogeografía y ecología de los peces de aguas continentales de la Argentina y consideraciones sobre las aguas ictiólicas de América del Sur. Ecosur, 2, 1-122.

Rosen, B. R. (1988). From fossils to earth history: Applied historical biogeography. En A. A. Myers y P. S. Giller (Eds.), Analytical biogeography (pp. 437-481). London: Chapman and Hall.

Van Jaarsveld, A. S., Freitag, S., Chown, S. L., Muller, C., Koch, S., Hull, H., et al. (1998). Biodiversity assessment and conservation strategies. Science, 279, 2106-2108 